\title{
Embolia de dióxido de carbono
}

\section{Carbon dioxide embolism}

Ruth Köhnenkampf MD. ${ }^{1}$

\begin{abstract}
Carbon dioxide $\left(\mathrm{CO}_{2}\right)$ embolism is a complication of laparoscopic surgery that, although often does not have adverse sequelae, can be fatal. This is due to the fact that when $\mathrm{CO}_{2}$ is injected into the blood vessels, the bubbles impede blood flow, which clinically expresses as: decreased stroke volume, hypoxemia, sudden fall or sudden increase in expired $\mathrm{CO}_{2}$, bradycardia, hypotension, dyspnea, cyanosis, arrhythmias, bilateral mydriasis, murmur in a mill wheel at auscultation and cardiovascular collapse with cardiorespiratory arrest. In this article we will present physiology of venous embolism, diagnosis, syntoms, treatment and prevention.
\end{abstract}

\section{RESUMEN}

La embolia por dióxido de carbono $\left(\mathrm{CO}_{2}\right)$ es una complicación de la cirugía laparoscópica que, aunque a menudo no presenta secuelas adversas, puede ser fatal. Esto se debe a que al inyectar $\mathrm{CO}_{2}$ en los vasos sanguíneos las burbujas impiden el flujo de sangre, lo que clínicamente se expresa como: disminución del volumen sistólico, hipoxemia, caída repentina o aumento súbito del $\mathrm{CO}_{2}$ espirado, bradicardia, hipotensión, disnea, cianosis, arritmias, midriasis bilateral, soplo en rueda de molino a la auscultación y al colapso cardiovascular con paro cardiorrespiratorio. En este trabajo presentaremos fisiología del embolismo venoso, cuadro clínico, diagnóstico, tratamiento y formas de prevenir que ocurra este evento.

\section{Key words:}

Carbon Dioxide, embolism, laparoscopy

\section{Palabras clave:}

Dióxido de carbono, embolia, laparoscopía

Anestesiólogo Servicio Viña del Mar/Quillota, Clínica Reñaca, Vigilius.

Fecha recepción: 24 de julio de 2019

Fecha de aceptación: 09 de agosto de 2019

Orcid

https://orcid.org/0000-0003-1837-5014

Correspondencia:

Ruth Köhnenkampf Cid

Email: rakohnen@me.com 


\section{Introducción}

a embolia por dióxido de carbono $\left(\mathrm{CO}_{2}\right)$ es una complicación rara de la cirugía laparoscópica[1],[2], ocurriendo en 15 por 100.000 casos por año, en 0,0014-0,6\% de las laparoscopías. Se cree que la embolia clínicamente significativa se produce en menos del $0,6 \%$ de las operaciones y se asocia con una tasa de mortalidad del $28 \%[2],[3],[4],[5]$. Se desconoce la incidencia en niños[2]. La gravedad del cuadro clínico, depende de la velocidad y el volumen de la entrada de gas[6]. Aunque a menudo sin secuelas adversas, estos émbolos son potencialmente fatales[2]. Los estudios realizados en humanos y en animales muestran una embolia gaseosa en aproximadamente la mitad de los casos de cirugía laparoscópica, según lo detectado por la ecocardiografía transesofágica[7],[8]. El mecanismo principal que conduce al desarrollo de émbolos es la colocación de la aguja de Veress directamente en una vena $u$ órgano parenquimatoso, pero también pueden ingresar pequeñas cantidades de gas a través de una abertura en cualquier vaso sanguíneo lesionado, ya sea en la pared abdominal o en el sitio operatorio o por la disección de estructuras altamente vascularizadas. Por lo tanto, no es sorprendente que el $60 \%$ de los casos sintomáticos ocurran durante la insuflación inicial, ocurriendo generalmente en los primeros 5 a 8 minutos después del inicio de la insuflación de $\mathrm{CO}_{2}[2],[9],[4],[3],[10],[5]$.

La presencia intravascular de pequeñas cantidades de gas soluble como el $\mathrm{CO}_{2}$ probablemente se produce con frecuencia sin tener ninguna consecuencia clínica, mientras que grandes cantidades de un gas soluble o pequeñas cantidades de un gas insoluble (aire, óxido nitroso) pueden causar la muerte[4]. El gas más utilizado para los procedimientos laparoscópicos es el $\mathrm{CO}_{2}$, es más, se recomienda estrictamente su uso en cirugía laparoscópica, es decir, no utilizar aire, para reducir la incidencia de embolias[11]. Esto se debe a que es un gas inerte barato, no inflamable y tiene la ventaja adicional de estar fácilmente disponible. Dado que el $\mathrm{CO}_{2}$ tiene un alto coeficiente de solubilidad de Ostwald en sangre y tejido $(L=0,54 \mathrm{ml}$ por $\mathrm{ml}$ de sangre total), y dado que la sangre tiene una alta capacidad amortiguadora para el $\mathrm{CO}_{2}$, las embolias de $\mathrm{CO}_{2}$ se absorben rápidamente si ocurren[8]. Este gas se usa, además, en la endoscopia gastrointestinal. Pese a lo descrito previamente, la alta solubilidad del $\mathrm{CO}_{2}$ no confiere necesariamente un efecto protector contra las embolias coronarias o cerebrales[12]. La persistencia del $\mathrm{CO}_{2}$ puede contribuir a la disfunción transitoria de órgano blanco e incluso llegar al paro cardíaco, aunque la eliminación del gas en cuestión de minutos no debería provocar un colapso cardiovascular sostenido en el contexto de una reanimación cardiopulmonar efectiva. En un estudio en perros[13], la magnitud de las perturbaciones fisiológicas causadas por el $\mathrm{CO}_{2}$ fue 6,5 veces menor que la del aire. Estas diferencias se atribuyeron a la alta solubilidad del $\mathrm{CO}_{2}$, la capacidad amortiguadora de la sangre para el $\mathrm{CO}_{2}$ y la rápida excreción de $\mathrm{CO}_{2}$ a través de los pulmones. Si bien el $\mathrm{CO}_{2}$ podría tener una "ventana terapéutica" mayor que la del aire, ambos gases pueden causar lesiones con embolia masiva. Las embolias pequeñas crean un riesgo significativamente mayor en presencia de derivaciones de derecha a izquierda[12]. La dosis de gas que conduce a complicaciones clínicas o la muerte en bolo o la administración de infusión continua solo se ha evaluado para diferentes gases, en experimentos con animales. Las dosis máximas toleradas, la DL50 para una inyección de bolo venoso de $\mathrm{CO}_{2}$ es de $25,0 \pm 1,7 \mathrm{ml} / \mathrm{kg}$ o para un humano de 70 $\mathrm{kg}$ sería de $1.750 \mathrm{ml}$ de $\mathrm{CO}_{2}[4],[5]$. Graff et al.[14] encontraron que se produjo hipotensión precipitada con una dosis endovenosa de $\mathrm{CO}_{2}$ de aproximadamente $30 \mathrm{ml} / \mathrm{kg}$ en perros.

También está descrita la existencia de la embolia paradójica de $\mathrm{CO}_{2}$, que es un evento extremadamente raro, pero puede tener consecuencias desastrosas y fatales. Se ha sugerido que la causa es la derivación intracardiaca de derecha a izquierda (principalmente debido a fosa oval permeable) o extracardíaca de gas venoso embolia[3]. Aunque el pulmón se considera un filtro fisiológico efectivo para los émbolos pulmonares, la capacidad de filtrado puede verse interferida por una cantidad abrumadora de gas en la circulación pulmonar y el uso de diversos agentes anestésicos, especialmente fentanilo y ketamina[15].

El objetivo de este artículo es hacer una descripción de la embolia de $\mathrm{CO}_{2}$ y de sus posibles formas de manejo, orientado a anestesiólogos y cirujanos. La información disponible respecto a este tema, se basa principalmente en reportes de caso. Queremos aclarar que este trabajo se trata exclusivamente de embolia de $\mathrm{CO}_{2}$, excluyéndose a otros tipos de embolias aéreas.

\section{Fisiología del embolismo venoso del dióxido de carbono}

Al inyectar $\mathrm{CO}_{2}$ en los vasos sanguíneos, el gas se desplaza en lugar de mezclarse porque inicialmente mantiene una interfase con la sangre, produciéndose un fenómeno de "bloqueo de gas" en el que las 
burbujas de $\mathrm{CO}_{2}$ impiden el flujo de sangre. Cuando este "bloqueo de gas" se ubica en el árbol arterial pulmonar, puede producirse la obstrucción del flujo sanguíneo. Cuando el bloqueo tiene lugar en el ventrículo derecho o la circulación pulmonar, puede disminuir el flujo de sangre hacia el ventrículo izquierdo, lo que resulta en una hipotensión sistémica. Además, la hipotensión puede ocurrir cuando más del 65\% del lecho vascular pulmonar está obstruido por las burbujas de $\mathrm{CO}_{2}$, aumentando el espacio muerto y disminuyendo el flujo sanguíneo pulmonar desde el lado derecho hacia el lado izquierdo del corazón. Las burbujas de $\mathrm{CO}_{2}$ también obstruyen el intercambio de gases en la interfaz alveolar-arterial, lo que conduce a una derivación fisiológica, dependiendo el grado de la gravedad de la embolia pulmonar. La hipotensión y la derivación fisiológica conducen a la hipoxemia. Además, la hipercarbia y la acidemia acompañante desplazan la curva de disociación de hemoglobina hacia la derecha, descargando oxígeno a nivel tisular y reduciendo aún más la tensión arterial del oxígeno[5].

\section{Diagnóstico}

El embolismo venoso de dióxido de carbono es un riesgo reconocido para la vida del paciente, por ello es fundamental un diagnóstico precoz y certero. Su presentación clínica varía de asintomática a compromiso neurológico, colapso cardiovascular o incluso muerte, dependiendo de la tasa y el volumen de atrapamiento de gases y la condición basal del paciente[3]. El diagnóstico precoz de la embolia gaseosa venosa es difícil, y la única evidencia concluyente de tal evento es la aspiración de gas desde la vasculatura o el corazón, que no siempre es práctico[5].

En un estudio de Mayer[5] en cerdos con infusión de $\mathrm{CO}_{2}$ endovenosa, todos los animales experimentaron hipertensión pulmonar, disminución del volumen sistólico, hipoxemia, hipercarbia y acidemia. Lo interesante de este estudio es que comparó las manifestaciones clínicas con una baja y alta tasa de infusión de $\mathrm{CO}_{2}$ y vio que los del primer grupo presentaron hipertensión sistémica y los del segundo grupo no. Además, los niveles de $\mathrm{CO}_{2}$ al final de la espiración, disminuyeron brevemente, luego aumentaron en todos los casos. Los animales que murieron presentaron hipotensión severa e hipoxemia, siendo signos predictores de casos potencialmente fatales. Los tres mecanismos posibles de muerte fueron la entrada de gas y la oclusión de la circulación coronaria, el gas atrapado en el ventrículo en sí mismo, lo que ocasiona un fallo de la acción de bombeo cardíaco y una embolia cerebral.
En humanos, una caída repentina de $\mathrm{EtCO}_{2}$ es un signo importante para la detección temprana del embolismo venoso de $\mathrm{CO}_{2}$, que se debe a la reducción de la perfusión pulmonar y al aumento del espacio muerto alveolar. Esto es primordial, sobre todo si el cambio de $\mathrm{EtCO}_{2}$ es durante la insuflación en la cirugía laparoscópica[1]. En relación a la clínica, se han descrito los siguientes síntomas: caída en saturación de oxígeno, bradicardia, $\mathrm{pCO}_{2}$ elevada, compromiso hemodinámico (hipotensión), disnea, cianosis, arritmias, midriasis súbita bilateral[16], soplo en rueda de molino a la auscultación (patognomónico), aumento repentino inicial en la concentración de $\mathrm{CO}_{2}$ al final de la espiración, que disminuye más tarde debido al colapso cardiovascular, aumento de presión venosa central, aspiración de burbujas en cateterismo venoso, en ETE se ha visto burbujas en carótida, cavidades cardiacas derechas e izquierdas (embolia paradójica), TAC con múltiples émbolos en arterias y venas cerebrales y cardíacas; además, paro cardiorrespiratorio, con resultado fatal[3],[11],,[17],[4],[6],[18]. Los cambios en el ECG no son lo suficientemente específicos ni lo suficientemente sensibles como para ser útiles en la detección del embolismo venoso de $\mathrm{CO}_{2}$ temprano. La presión arterial pulmonar elevada (PAP) es un indicador sensible de la embolia gaseosa venosa. La hipercarbia grave puede alertar al anestesiólogo sobre la posibilidad de embolia venosa de $\mathrm{CO}_{2}$. Parte del $\mathrm{CO}_{2}$ infundido que no es eliminado por los pulmones puede disolverse en el plasma y depositarse en el esqueleto muscular. Esta cantidad de $\mathrm{CO}_{2}$ se excreta mucho después de la infusión de $\mathrm{CO}_{2}$, y puede explicar los efectos hemodinámicos prolongados durante el período de recuperación. La hipoxemia inmediata puede ser el resultado de una embolia gaseosa venosa, pero primero deben excluirse otras causas potenciales: malposición del tubo endotraqueal, neumotórax e hipovolemia. Es muy probable que sea el resultado de una distribución de la relación ventilación-perfusión no coincidente, o derivación fisiológica debido a las burbujas de $\mathrm{CO}_{2}$ que obstruyen la interfaz alveolar-arterial. La hipotensión, cuando se asocia con una hipoxemia grave, fue un predictor de embolismo venoso de $\mathrm{CO}_{2}$ potencialmente mortal[5]. La tríada de disminución súbita de $\mathrm{EtCO}_{2}$, hipotensión sistólica y desaturación sin cambio en la presión de la vía aérea hace sospechar como causa una embolia de $\mathrm{CO}_{2}$.

En la embolia paradójica, la expresión clínica es similar a la antes mencionada: caída abrupta $\mathrm{EtCO}_{2}$ tras insuflación, la saturación de oxígeno disminuye rápidamente, la presión arterial sistólica baja, taquicardia sinusal con depresión significativa del ST, aci- 
dosis, elevación de la $\mathrm{PaCO}_{2}$ y caída de $\mathrm{PaO}_{2}[3]$.

El número de eventos clínicos y complicaciones se relaciona directamente con el tipo y la cantidad de gas insuflado[4]. Al analizar la fisiopatología del embolismo de $\mathrm{CO}_{2}$ a través de modelos animales, podemos entender mejor que hay un rango de dosis que lleva a cambios a nivel de circulación pulmonar, condición ácido base y falla multiorgánica en una etapa final, lo que define finalmente la sintomatología. En un modelo de lechón, la infusión continua de 0,75 $\mathrm{ml} / \mathrm{kg} / \mathrm{min}$ de dióxido de carbono dio lugar a hipertensión pulmonar, acidosis e hipoxemia; sin embargo, no se produjeron muertes hasta que la velocidad de infusión se incrementó a 1,2 ml/kg/min[5].

En relación a la confirmación diagnóstica, tenemos 3 opciones: aspiración de gas de un catéter venoso central, ecocardiografía transtorácica[8] y la ecocardiografía transesofágica (ETE) (Figura 1). Esta última es el método más confiable para el embolismo por $\mathrm{CO}_{2}$, siendo considerado el gold standard[19]. El ecocardiograma transesofágico durante el colapso cardiovascular en cirugía no cardíaca es una modalidad de imagen valiosa que puede diagnosticar con eficacia la hipovolemia, la disfunción cardíaca, la isquemia/infarto de miocardio y el tromboembolismo pulmonar en fase sólida[18]. La ETE puede detectar gas intravenoso en la vena cava inferior, el ventrículo derecho y la arteria pulmonar, pero no se usa de forma rutinaria en el monitoreo intraoperatorio debido a su alto costo y complejidad técnica[1]. Estudios recientes, realizados en pacientes adultos, han demostrado una alta prevalencia de embolia gaseosa durante los procedimientos laparoscópicos cuando se utiliza la ecocardiografía transesofágica como herramienta de diagnóstico. A modo de ejemplo, podemos destacar que todas las pacientes que se sometieron a una histerectomía laparoscópica total sufrieron una embolia de gas en la aurícula derecha, y un tercio de ellos tuvieron una embolia de gas de alto grado (definido por Kim et al. como gas detectable que exce- de la mitad del diámetro de la aurícula derecha, el ventrículo derecho o el tracto de salida del ventrículo derecho)[20]. Cuando se estudia con ecocardiografía transesofágica, el $68 \%$ de los pacientes asintomáticos tienen burbujas de $\mathrm{CO}_{2}$ en la cámara derecha del corazón durante la colecistectomía laparoscópica[4]. Otro ejemplo, es que el $17 \%$ de los pacientes sometidos a una prostatectomía radical laparoscópica sufren una embolia de gas auricular derecho[4]. Lo anterior nos lleva a plantear la siguiente pregunta: si el embolismo de $\mathrm{CO}_{2}$ es tan frecuente, ¿cuándo se vuelve clínicamente significativo?

La embolia gaseosa clínicamente relevante es muy rara, pero si ocurre, puede ser una complicación fatal. No se conoce la verdadera incidencia de embolia gaseosa clínicamente inaparente[9]. Un estudio en cerdos hizo un análisis postoperatorio con ETE, evidenciando embolia aérea en 19 de 26 casos. Sin embargo, de los 19 casos en que se observaron embolias, 18 de los sujetos no experimentaron ningún cambio hemodinámico significativo. Estos datos sugieren que el embolismo de burbujas pequeñas no es probable que cause consecuencias hemodinámicas y se debe absorber rápidamente debido a la solubilidad del $\mathrm{CO}_{2}$ en el plasma. Sin embargo, las burbujas grandes pueden provocar un bloqueo de gas y pueden conducir a una obstrucción del flujo vascular pulmonar[21].

En relación a la ETE, Schmandra[10] describió en el año 2002 una clasificación que ha sido utilizada en estudios posteriores, para ser utilizada en embolismo aéreo. Esta Tabla 1 fue descrita en estudio de cerdos con hepatectomía laparoscópica, por lo que se utilizó para embolia de $\mathrm{CO}_{2}$.

\section{Factores de riesgo}

Durante la laparoscopia, la insuflación de presión positiva de la cavidad peritoneal utilizando dióxido de carbono es necesaria para permitir la visualización.

Tabla 1. Clasificación de Schmandra para embolismo gaseoso utilizando ecocardiografía transesofágica en 2
dimensiones

AD: aurícula derecha; VD: VD TSVD: tracto de salida ventrículo derecho. 
Esta inyección es el principal factor de riesgo de embolia de $\mathrm{CO}_{2}$, dado que la causa más común es la inyección inadvertida de dióxido de carbono en una vena grande u órgano sólido durante la insuflación peritoneal inicial[2]. Debemos considerar también como predisponentes a embolia la posición de Trendelenburg (dado que la presión venosa es baja y el $\mathrm{CO}_{2}$ puede entrar fácilmente en las venas)[22] 95\% $\mathrm{Cl}$, las presiones intraabdominales $>15 \mathrm{mmHg}$ [23] y cuando se usa anestesia de baja presión venosa central para minimizar la hemorragia, dado que existe un aumento teórico del riesgo de embolia de $\mathrm{CO}_{2}$ a presiones de insuflación que exceden la PVC, lo que causa un gradiente de presión en la circulación veno$\mathrm{sa}[21],[24]$.

Además, el uso de óxido nitroso para anestesia, puede aumentar el riesgo de desarrollar embolia gaseosa durante la laparoscopia[9].

En la literatura, los casos descritos de embolismo de $\mathrm{CO}_{2}$ corresponden a las siguientes cirugías: prostatectomía radial, histerectomía[20], nefrectomía[3], hepatectomía[21], colangioscopia endoscópica directa[17], colecistectomía con resección de quistes hepáticos[8], hepatectomía[10], cirugías ginecológicas[25], cirugía de fosa posterior[26] y adherenciolisis[18]. En cirugía pediátrica se describió un embolismo con dióxido de carbono durante colecistectomía laparoscópica debido a una vena paraumbilical patente[2].

\section{Prevención}

El embolismo de dióxido de carbono es una complicación reconocida de la cirugía laparoscópica, aunque el riesgo para el paciente puede minimizarse si el equipo quirúrgico se da cuenta del problema, se realiza un monitoreo intraoperatorio continuo del $\mathrm{CO}_{2}$ tidal y se utiliza una técnica abierta para el acceso inicial al peritoneo[2]. En la práctica clínica, hay pocas opciones técnicas disponibles para reducir el riesgo de embolia gaseosa. Por lo tanto, es muy importante que el cirujano que crea el neumoperitoneo tenga experiencia en técnicas de acceso laparoscópico[9].

La Asociación Europea de Cirugía Endoscópica en su guía de práctica clínica[9] da recomendaciones en relación al neumoperitoneo para cirugía laparoscópica:

- Antes de la operación, todos los pacientes deben ser evaluados en cuanto a la presencia de comorbilidad cardíaca, pulmonar, hepática, renal o vascular.

- $\quad$ Si se realizan medidas perioperatorias y técnicas quirúrgicas adecuadas, no hay razón para contra-

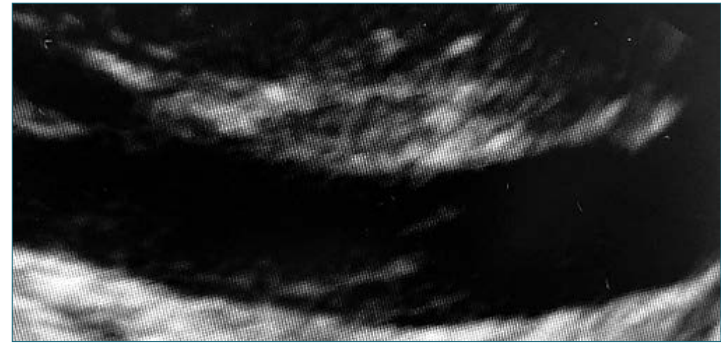

Figura 1. Ecocardiograma transtorácico subcostal con visión de vena cava inferior con burbujas de $\mathrm{CO}_{2}$.

indicar el neumoperitoneo en pacientes con peritonitis o neoplasia intraabdominal.

- Durante la laparoscopia, el monitoreo de la concentración final de $\mathrm{CO}_{2}$ tidal es obligatorio.

- Los datos disponibles sobre la técnica de aguja cerrada (y la técnica de acceso abierto) no nos permiten favorecer principalmente el uso de ninguna de estas técnicas.

- Se recomienda utilizar la presión intraabdominal más baja que permita una exposición adecuada del campo operatorio, en lugar de utilizar una presión de rutina.

- En pacientes con función cardíaca, pulmonar o renal limitada, la elevación de la pared abdominal combinada con neumoperitoneo a baja presión podría ser una alternativa.

- En pacientes con enfermedades cardiopulmonares, se recomienda la monitorización intraoperatoria y posoperatoria de los gases en sangre arterial.

- Cuando se usa la aguja de Veress, se recomienda que la insuflación inicial se realice con flujo bajo para evitar un bolo grande[2].

- El cirujano debe estar preparado para controlar rápidamente la hemorragia venosa grande[2].

Existe un nivel I de evidencia de que la técnica de Hasson para el establecimiento de neumoperitoma es más segura que la técnica de la aguja de Veress; la incidencia de embolia gaseosa informada por una revisión sistemática fue del 0,001\% (7/489.000 casos) para la aguja de Veress, mientras que en más de 12.000 casos no se presentaron complicaciones con la técnica de Hasson. Por lo tanto, se recomienda utilizar este último en lugar de la aguja de Veress en todos los casos[4]. Se puede suponer que los trocares romos reducen el riesgo de punción accidental del vaso[9].

Debido a la baja incidencia de embolias gaseosas clínicamente relevantes, no se puede recomendar para 
la rutina clínica la monitorización invasiva avanzada (ecografía transesofágica)[9]. En relación a esto, Pandia y cols., en el año 2019, publicaron un estudio[26] en donde se comparó la significancia clínica de embolias detectadas con ETE con y sin cambios en $\mathrm{ETCO}_{2}$. En aquellos pacientes en que no hubo cambios en el $\mathrm{ETCO}_{2}$, no se apreciaron cambios en la hemodinamia, mientras que en aquellos que sí presentaron una caída en la capnografía, presentaron una incidencia de $20 \%$ de taquicardia y $30 \%$ de hipotensión asociadas. Es por esto que la conclusión de los autores es que el monitoreo de $\mathrm{ETCO}_{2}$ es suficiente para detectar embolias gaseosas clínicamente significativas. Además, el diagnóstico de un episodio transitorio y clínicamente no significativo de embolia, cuando sólo unas pocas burbujas de gas atraviesan la pantalla del ecocardiógrafo, requiere un enfoque continuo en el monitor, que no siempre es práctico para un anestesiólogo ocupado con muchas otras responsabilidades.

Hay que considerar el riesgo de embolia de aire al no purgar adecuadamente el sistema con gas $\mathrm{CO}_{2}$ antes de la inserción de los trócares en cirugía laparoscópica[8].

\section{Tratamiento}

Si se sospecha una embolia, se deben realizar inmediatamente una serie de medidas que incluyen[4],[2]:

- Deflación del neumoperitoneo.

- Colocación del paciente en la posición de Durant (decúbito lateral izquierdo y Trendelemburg) que permite que el gas suba hacia el vértice del ventrículo derecho, lejos del tracto de salida del ventrículo derecho, evitando así su entrada en la arteria pulmonar. La posición de Trendelenburg por sí sola también es suficiente ya que tiene el mismo efecto.

- La hiperventilación y la administración de oxígeno al $100 \%$ ayudan a la rápida eliminación de $\mathrm{CO}_{2}$.

- Se debe realizar una resucitación cardiopulmonar agresiva, enfocada en manejo de hipotensión persistente o arritmia.

- Instalación de un catéter venoso central para aspirar el gas.

- El oxígeno hiperbárico es menos útil que para las embolias del aire porque el $\mathrm{CO}_{2}$ es más soluble y existe un gradiente de alta presión entre la sangre y las burbujas de $\mathrm{CO}_{2}$ (más de $600 \mathrm{~mm} \mathrm{Hg}$ ), lo que fomenta la reabsorción. El uso de esta técnica solo está justificado si la cámara está disponible dentro de un corto período de tiempo y si es po- sible continuar reviviendo al paciente durante la sesión terapéutica de oxígeno. Si está disponible, dicho tratamiento a menudo consistirá en la compresión del paciente a $285 \mathrm{kPa}$ (es decir, 2,8 atm abs) respirando oxígeno. El propósito es: 1) reducir el tamaño de la burbuja intravascular mediante la compresión de la fase gaseosa de acuerdo con la Ley de Boyles; 2) maximizar los gradientes de presión parciales de gas de las burbujas y los tejidos a la sangre, aumentando así la velocidad de eliminación de la burbuja y 3) introducir el sistema de hiperoxia en un tejido por lo demás hipóxico. El primer objetivo se alcanza casi de inmediato mediante la compresión, independientemente de la mezcla de respiración utilizada, mientras que la respiración de oxígeno lograría los dos últimos objetivos. Una presión arterial parcial de oxígeno superior a $2.000 \mathrm{mmHg}$ se alcanza fácilmente. La hiperoxia también aumenta la distancia de difusión de oxígeno en los tejidos y contrarresta el insulto embólico a la microvasculatura. El oxígeno hiperbárico es el tratamiento de elección para la embolia gaseosa arterial. El tiempo es importante, pero el tratamiento tardío en una cámara hiperbárica aún puede estar indicado para mejorar la condición del paciente y reducir las secuelas neurológicas a largo plazo[8].

La terapia de oxígeno hiperbárico es la primera línea de tratamiento para el embolismo arterial del gas[8]. Tres casos por 100.000 pueden necesitar tratamiento hiperbárico para embolias gaseosas y solo 7/4.727.496 casos se describen como causados por cirugía laparoscópica con insuflación intraperitoneal de $\mathrm{CO}_{2}[27]$.

Hay un reporte de caso en que además de reanimación cardiopulmonar avanzada, se utilizó como tratamiento oxígeno hiperbárico, con resultado favorable para el paciente[8].

\section{Conclusión}

La rara incidencia de embolia venosa de $\mathrm{CO}_{2}$ puede contribuir a su diagnóstico erróneo o retraso en el diagnóstico a menos que el índice de sospecha sea alto[5]. Es por esto que es fundamental que el equipo quirúrgico sospeche una embolia durante la laparoscopía cuando el paciente desarrolle hipotensión severa repentina e inexplicable, cambio súbito de $\mathrm{EtCO}_{2} \mathrm{O}$ paro cardíaco durante o inmediatamente después de la laparoscopía, dado que el resultado puede ser fatal si no se trata de inmediato. Además, es importante 
identificar parámetros clínicos simples, no invasivos, pero fácilmente disponibles que puedan indicar embolia venosa de $\mathrm{CO}_{2}$ durante el inicio del neumoperitoneo[5]. La baja presión intraabdominal, las bajas tasas de insuflación, así como una técnica quirúrgica cuidadosa pueden reducir la incidencia de embolia gaseosa[9].

Quiero además agradecer en forma especial a la Dra. María Carolina Cabrera y Dr. Fernando Aranda por brindar su tiempo y esfuerzo para evaluar este trabajo. Gracias por su destacada colaboración y sus valiosos aportes.

\section{Referencias}

1. Burcharth J, Burgdorf S, Lolle I, Rosenberg J. Successful Resuscitation After Carbon Dioxide Embolism During Laparoscopy. Surgical Laparoscopy, Endoscopy \& Percutaneous Techniques [Internet]. Ovid Technologies (Wolters Kluwer Health); 2012 Jun;22(3):e164-e167. Available from: http://dx.doi.org/10.1097/ sle.0b013e31825150a9.

2. Mattei P, Tyler DC. Carbon dioxide embolism during laparoscopic cholecystectomy due to a patent paraumbilical vein Journal of Pediatric Surgery. marzo de 2007;42(3):570-2. https://doi.org/10.1016/j.jpedsurg.2006.10.051.

3. Huang $Y-Y$, Wu H-L, Tsou M-Y, Zong H-J, Guo W-Y, Chan $\mathrm{K}-\mathrm{H}$, et al. Paradoxical Carbon Dioxide Embolism During Pneumoperitoneum in Laparoscopic Surgery for a Huge Renal Angiomyolipoma. Journal of the Chinese Medical Association. abril de 2008;71(4):214-7. https://doi.org/10.1016/S17264901(08)70107-2.

4. Gutt CN, Oniu T, Mehrabi A, Schemmer P, Kashfi A, Kraus $T$, et al. Circulatory and respiratory complications of carbon dioxide insufflation. Dig Surg. 2004;21(2):95-105. https:// doi.org/10.1159/000077038 PMID:15010588

5. Mayer KL, Ho HS, Mathiesen KA, Wolfe BM. Cardiopulmonary responses to experimental venous carbon dioxide embolism. Surgical Endoscopy. agosto de 1998;12(8):1025130. https://doi.org/10.1007/ s004649900773.

6. Shen JC, Ji MH, Yang C, Wang $X$, Yang JJ. Severe carbon dioxide embolism during laparoscopic kidney resection. Acta Anaesthesiologica Scandinavica. Wiley; 2011 Mar 11;55(4):505-505. http://dx.doi.org/10.1111/j.13996576.2011.02408.x

7. Hong J-Y, Kim WO, Kil HK. Detection of Subclinical CO2 Embolism by Transesophageal Echocardiography During Laparoscopic Radical Prostatectomy. Urology. marzo de 2010;75(3):581-4 https://doi.org/10.1016/j.urology.2009.04.064.

8. Kjeld T, Hansen EG, Holler NG, Rottensten $\mathrm{H}$, Hyldegaard $\mathrm{O}$, Jansen EC. Resuscitation by hyperbaric exposure from a venous gas emboli following laparoscopic surgery. Scand J Trauma Resusc Emerg Med. 2012 Aug;20(1):51. https://doi. org/10.1186/1757-7241-20-51 PMID:22862957

9. Neudecker J, Sauerland S, Neugebauer E, Bergamaschi R, Bonjer HJ, Cuschieri A, et al. The European Association for Endoscopic Surgery clinical practice guideline on the pneumoperitoneum for laparoscopic surgery. Surgical Endoscopy. julio de 2002;16(7):1121-43. https://doi. org/10.1007/s00464-001-91667.

10. Schmandra TC, Mierdl S,
Bauer H, Gutt C, Hanisch E. Transoesophageal echocardiography shows high risk of gas embolism during laparoscopic hepatic resection under carbon dioxide pneumoperitoneum. British Journal of Surgery. julio de 2002;89(7):870-6. https:// doi.org/10.1046/j.13652168.2002.02123.x.

11. Hann A, Zizer E, Egger K, Allescher $\mathrm{H}-\mathrm{D}$, Meining A. Fatal outcome due to $\mathrm{CO} 2$ emboli during direct cholangioscopy. Gut. BMJ; 2017 Mar 30;67(8):1378-9. http://dx.doi.org/10.1136/gutjnl-2017-313988

12. Fischler M. Occurrence of a carbon dioxide embolism during laparoscopic pyloromyotomy in a small child: several unresolved questions. Canadian Journal of Anesthesia/Journal canadien d'anesthésie. febrero de 2011;58(2):226-7. https://doi. org/10.1007/s12630-010-9425-y.

13. Steffey EP, Johnson BH, Eger El 2nd. Nitrous oxide intensifies the pulmonary arterial pressure response to venous injection of carbon dioxide in the dog. Anesthesiology. 1980 Jan;52(1):52-5. https:// doi.org/10.1097/00000542198001000-00011 PMID:7352646

14. Graff TD, Arbegast NR, Phillips OC, Harris LC, Frazier TM. Gas embolism: A comparative study of air and carbon dioxide as embolic agents in the systemic venous system. American Journal of Obstetrics and Gynecology. 
agosto de 1959;78(2):259-65. https://doi.org/10.1016/00029378(59)90169-3.

15. Yahagi $N$, Furuya $H$, Sai $Y$, Amakata Y. Effect of halothane, fentanyl, and ketamine on the threshold for transpulmonary passage of venous air emboli in dogs. Anesth Analg. 1992 Nov;75(5):720-3. https:// doi.org/10.1213/00000539199211000-00011 PMID:1416124

16. Cottin V, Delafosse B, Viale J-P. Gas embolism during laparoscopy A report of seven cases in patients with previous abdominal surgical history. Surgical Endoscopy. Springer Science and Business Media LLC; 1996 Feb 1;10(2):166-9. http://dx.doi. org/10.1007/s004649910038.

17. Kondo H, Naitoh I, Nakazawa T, Hayashi K, Nishi Y, Umemura $S$, et al. Development of fatal systemic gas embolism during direct peroral cholangioscopy under carbon dioxide insufflation. Endoscopy. 16 de junio de 2016;48(S 01):E215-6. https:// doi.org/10.1055/s-0042-109056.

18. Patel JH, Szymanski TJ, Metzler EC, Worthington AH, Body SC, Lu JT. Rescue Transesophageal Echocardiography for the Diagnosis and Management of Paradoxical Carbon Dioxide Embolism With Hemodynamic Compromise During Laparoscopic Surgery. Journal of
Cardiothoracic and Vascular Anesthesia. junio de 2013;27(3): e23-4. https://doi.org/10.1053/j. jvca.2012.09.020.

19. Mirski MA. Diagnosis and Treatment of Vascular Air Embolism. Spinal fusion. 2007;106(1):14. https://doi. org/10.1097/00000542200701000-00026.

20. Venous Air Embolism during Total Laparoscopic HysterectomyComparison to Total Abdominal Hysterectomy. 2009;111(1):5.

21. Jayaraman $S$, Khakhar $A$, Yang $H$, Bainbridge D, Quan D. The association between central venous pressure, pneumoperitoneum, and venous carbon dioxide embolism in laparoscopic hepatectomy. Surgical Endoscopy. octubre de 2009;23(10):236973. https://doi.org/10.1007/ s00464-009-0359-9.

22. Hong JY, Kim JY, Choi YD, Rha KH, Yoon SJ, Kil HK. Incidence of venous gas embolism during robotic-assisted laparoscopic radical prostatectomy is lower than that during radical retropubic prostatectomy ++ This article was presented as one of the best papers in the 13th Asian Australian Congress of Anesthesiologists (Fukuoka, Japan, June 1-5, 2010). British Journal of Anaesthesia. Elsevier BV; 2010 Dec;105(6):777-81. http://dx.doi. org/10.1093/bja/aeq247
23. Park EY, Kwon JY, Kim KJ. Carbon dioxide embolism during laparoscopic surgery. Yonsei Med J. 2012 May;53(3):459-66. https://doi. org/10.3349/ymj.2012.53.3.459 PMID:22476987

24. Bazin JE, Gillart T, Rasson P, Conio N, Aigouy L, Schoeffler P. Haemodynamic conditions enhancing gas embolism after venous injury during laparoscopy: a study in pigs. British Journal of Anaesthesia. mayo de 1997;78(5):570-5. https://doi. org/10.1093/bja/78.5.570.

25. Hynes SR, Marshall RL. Venous gas embolism during gynaecological laparoscopy. Can J Anaesth. 1992 Sep;39(7):7489. https://doi.org/10.1007/ BF03008249 PMID:1394772

26. Pandia MP, Bithal PK, Dash HH, Chaturvedi A. Comparative incidence of cardiovascular changes during venous air embolism as detected by transesophageal echocardiography alone or in combination with end tidal carbon dioxide tension monitoring. Journal of Clinical Neuroscience. septiembre de 2011;18(9):12069. https://doi.org/10.1016/j. jocn.2011.01.023.

27. Muth CM, Shank ES. Gas embolism. N Engl J Med. 2000 Feb;342(7):476-82. https://doi.org/10.1056/ NEJM200002173420706 PMID:10675429 